Nota de investigación

\title{
Efecto de bioestimulantes radiculares sobre el crecimiento en plantas de aguacate
}

\author{
Braulio Alberto Lemus-Soriano ${ }^{\S}$ \\ Eulalio Venegas-González \\ Marco Aurelio Pérez-López
}

Facultad de Agrobiología 'Presidente Juárez'-Universidad Michoacana de San Nicolás de Hidalgo. Paseo Lázaro Cárdenas esq. Berlín s/n, Col. Viveros, Uruapan, Michoacán, México. CP. 60170. (alberto.lemus@umich.mx; lalovenegas@prodigy.net.mx,map127@hotmail.com).

§Autor para correspondencia: alberto.lemus@umich.mx.

\section{Resumen}

Para un adecuado y exitoso establecimiento del cultivo de aguacate se necesitan plantas con un sistema radicular desarrollado y sano. Sin embargo, en los viveros comerciales de aguacate no se realizan manejos orientados a la mejora radicular. El objetivo del presente trabajo fue evaluar el efecto de bioestimulantes radiculares a base de microorganismos y ácidos orgánicos sobre el crecimiento, contenido de clorofila y micorrización en plantas de aguacate. Se tuvieron siete tratamientos, incluido un testigo. El diseño experimental fue completamente al azar, con ocho repeticiones. Las variables evaluadas fueron número de hojas, altura de la planta, longitud de la raíz principal, unidades SPAD, peso seco de la raíz y porcentaje de micorrización. En cada una de las variables, el mejor tratamiento fue la combinación Nutrisorb ${ }^{\circledR} \mathrm{L}+$ Biofit $^{\circledR} \mathrm{RTU}$, lo cual confirma que el uso de microorganismos y ácidos orgánicos favorece el crecimiento vegetativo y radicular, así como promueve una mayor micorrización.

Palabras clave: ácidos orgánicos, aguacate, microorganismos.

Recibido: agosto de 2021

Aceptado: septiembre de 2021 
El aguacate es uno de los cultivos de mayor importancia económica en el país, al mes de enero de 2020 la producción obtenida de aguacate en México fue de 206466 t, donde el estado Michoacán aportó 94.6\% (195366 t) al total nacional, lo que lo convierte el principal productor (SIAP, 2020).

En México la producción de planta de aguacate se basa principalmente en el uso de portainjertos originados por semilla; sin embargo, no se cuenta de planta certificada de aguacate, ya que aún no se dispone de la regla técnica respectiva que indique los procedimientos para producir planta en vivero, por lo tanto, el procedimiento para la propagación es variable (Campos-Rojas et al., 2012).

En cuanto a su manejo en el vivero, se fertilizan principalmente con fosfato diamónico (DAP-1846-00) así como aplicaciones de algunas otras fuentes de fertilizantes químicos (Ortíz-Estrella y Vázquez-Collado, 2008). Por otra parte, existen los bioestimulantes derivados de diversas sustancias y microorganismos que ayudan a mejorar el crecimiento de las plantas (Calvo et al., 2014; du Jardin, 2015). Asimismo, las raíces de las plantas influyen sobre las características físicas, químicas y biológicas de la rizosfera del suelo (Koo et al., 2005) y actúan directamente sobre los microorganismos por la secreción de distintos compuestos (Bais et al., 2006).

El uso de inoculantes microbiales como las rizobacterias, hongos endófitos y micorrícicos se ha incrementado en los últimos años con distintos fines (Hayat et al., 2010). Por lo que el objetivo propuesto fue evaluar el efecto de diferentes bioestimulantes sobre el desarrollo de plántulas de aguacate. El presente estudio se realizó en las instalaciones de la Facultad de Agrobiología 'Presidente Juárez' dependiente de la Universidad Michoacana de San Nicolás de Hidalgo, localizada en la ciudad de Uruapan, Michoacán en las coordenadas $19^{\circ} 23^{\prime} 41.375^{\prime \prime}$ latitud norte, $102^{\circ}$ 3' 30.192"' longitud oeste y una altitud de $1589 \mathrm{~m}$.

Se utilizaron plantas de un vivero comercial de la localidad de Tingambato, Michoacán. Estas tenían 15 días de injertadas con la variedad Hass en patrón criollo de la región y presentaban un porte visual homogéneo, posteriormente fueron trasladadas al área experimental (invernadero) dentro de las instalaciones de la Facultad de Agrobiología.

Se utilizó un diseño completamente al azar, con siete tratamientos y ocho repeticiones. La unidad experimental consistió en una planta de aguacate. Los productos evaluados fueron un testigo (control) y los bioestimulantes comerciales: Nutrisorb ${ }^{\circledR}$ L (ácidos carboxílicos, 11\%), Mycoroot ${ }^{\circledR}$ (Pisolithus tinctrius, $1 \times 10^{6} \mathrm{UFC} \mathrm{g}^{-1}$, Glomus intraradices, 1 x $10^{3} \mathrm{UFC} \mathrm{g}^{-1}$; Azospirillum

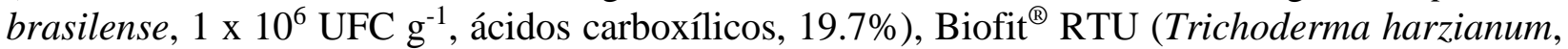
$1.35 \times 10^{5} \mathrm{UFC} \mathrm{g}^{-1}$, Penicillium bilaiae + Penicillium spp. + Paecilomyces lilacinus, $1.25 \times 10^{7}$ UFC g ${ }^{-1}$, Bacillus subtilis, $1.25 \times 10^{8} \mathrm{UFC} \mathrm{g}^{-1}$, Azospirillum brasilense, $1.25 \times 10^{5} \mathrm{UFC} \mathrm{g}^{-1}$, ácidos carboxílicos, $34 \%$ ) y Glumix ${ }^{\circledR}$ (Glomus spp. 1 x $10^{3}$ UFC g $^{-1}$ ) (Cuadro 1).

Cuadro 1. Tratamientos evaluados para determinar su efecto bioestimulante en plántulas de aguacate bajo condiciones de invernadero en Uruapan, Michoacán.

\begin{tabular}{|c|c|}
\hline Tratamiento & Dosis * \\
\hline A) Nutrisorb ${ }^{\circledR} \mathrm{L}$ & $3 \mathrm{ml}$ \\
\hline B) Mycoroot ${ }^{\circledR}$ & $5 \mathrm{~g}$ \\
\hline C) Biofit ${ }^{\circledR}$ RTU & $5 \mathrm{~g}$ \\
\hline D) Nutrisorb ${ }^{\circledR} L+$ Mycoroot $^{\circledR}$ & $3 \mathrm{ml}+5 \mathrm{~g}$ \\
\hline
\end{tabular}




\begin{tabular}{|c|c|}
\hline Tratamiento & Dosis * \\
\hline E) Nutrisorb ${ }^{\circledR} L+$ Biofit $^{\circledR}$ RTU & $3 \mathrm{ml}+5 \mathrm{~g}$ \\
\hline F) Glumix ${ }^{\circledR}$ & $5 \mathrm{~g}$ \\
\hline G) Testigo & Agua \\
\hline
\end{tabular}

* = dosis en un litro de agua por plántula por aplicación.

Las aplicaciones se realizaron en drench cada 21 días. En total nueve aplicaciones iniciando en octubre de 2019 y terminando en abril de 2020 donde se evaluaron las siguientes variables respuesta: número de hojas, altura de la planta, longitud de la raíz principal, unidades SPAD y peso seco de la raíz, además se determinó el porcentaje de micorrización. Con los datos obtenidos se realizó un análisis de varianza y una prueba de separación de medias de Tukey $\alpha=0.05$, con el programa estadístico Statistical Analysis System versión 9.0 (SAS, 2002). Todas las variables agronómicas evaluadas presentaron diferencias altamente significativas $(p \leq 0.01)$ para tratamientos en los análisis de varianza (Cuadro 2).

Cuadro 2. Respuesta de plántulas de aguacate a distintos tratamientos de bioestimulantes en Uruapan, Michoacán.

\begin{tabular}{|c|c|c|c|c|c|}
\hline Tratamientos & $\begin{array}{l}\text { Número } \\
\text { de hojas }\end{array}$ & $\begin{array}{l}\text { Contenido de clorofil } \\
\text { (unidades SPAD) }\end{array}$ & $\begin{array}{l}\text { a Altura de la } \\
\text { planta }(\mathrm{cm})\end{array}$ & $\begin{array}{l}\text { Longitud de } \\
\text { raíz }(\mathrm{cm})\end{array}$ & $\begin{array}{l}\text { Peso seco } \\
\text { de raíz }(\mathrm{g})\end{array}$ \\
\hline A) Nutrisorb ${ }^{\circledR} L$ & $40.12 \mathrm{~b}$ & $66.86 \mathrm{~b}$ & $51.7 \mathrm{bc}$ & $43.47 \mathrm{bc}$ & $8.62 \mathrm{~d}$ \\
\hline B) Mycoroot $^{\circledR}$ & $40 \mathrm{bc}$ & $72.37 \mathrm{ab}$ & $49.42 \mathrm{c}$ & $49.38 \mathrm{~b}$ & $12.75 \mathrm{bc}$ \\
\hline C) Biofit ${ }^{\circledR}$ RTU & $38.75 \mathrm{bc}$ & $70.47 \mathrm{ab}$ & $51.77 \mathrm{bc}$ & $45.12 \mathrm{bc}$ & $11.96 \mathrm{c}$ \\
\hline D) Nutrisorb ${ }^{\circledR} L+$ Mycoroot $^{\circledR}$ & $40.25 \mathrm{~b}$ & $68.56 \mathrm{ab}$ & $53.37 \mathrm{~b}$ & $49.38 \mathrm{~b}$ & $13.65 \mathrm{~b}$ \\
\hline E) Nutrisorb ${ }^{\circledR} \mathrm{L}+$ Biofit $^{\circledR}$ RTU & $53.87 \mathrm{a}$ & $75.48 \mathrm{a}$ & $58.58 \mathrm{a}$ & $63.23 \mathrm{a}$ & $24.5 \mathrm{a}$ \\
\hline F) Glumix ${ }^{\circledR}$ & $41.75 \mathrm{~b}$ & $65.25 \mathrm{~b}$ & $54.87 \mathrm{~b}$ & $47.5 \mathrm{~b}$ & $13.75 \mathrm{~b}$ \\
\hline G) Testigo & $32.62 \mathrm{c}$ & $55.65 \mathrm{c}$ & $41.91 \mathrm{~d}$ & $34.25 \mathrm{c}$ & $4.91 \mathrm{e}$ \\
\hline
\end{tabular}

Las medias agrupadas con la misma literal no difieren estadísticamente entre sí, según la prueba de Tukey $(p \leq 0.05)$.

En las pruebas de comparación de medias de Tukey para las variables se observó que la mezcla de Nutrisorb ${ }^{\circledR}$ L + Biofit ${ }^{\circledR}$ RTU presentó mayor número de hojas con una media de 57.87 hojas por planta. Esto coincide con González y Fuentes (2017) que evaluaron diferentes microorganismos los cuales produjeron efectos benéficos en el número de hojas para las plantas de girasol (Helianthus annuus L). Sakthiselvan et al. (2014) y han sugerido que los microrganismos pueden favorecer el crecimiento vegetal, ya que generan un efecto positivo sobre algunas propiedades químicas del suelo aumentando la solubilización de nutrientes y la capacidad de absorción de estos.

Es común que el contenido de clorofila se utilice en programas de manejo nutricional (Blasco et $a l ., 2010)$ y es una herramienta útil para dar seguimiento a la nutrición y con ello mejorar el rendimiento del cultivo (López-Bellido et al., 2004). El tratamiento de Nutrisorb ${ }^{\circledR}$ L + Biofit $^{\circledR}$ RTU presentó el mayor contenido de clorofila con 75.48 unidades SPAD. Los resultados obtenidos difieren de los encontrados por Arellano (2017) quien en el caso del contenido de clorofila en hojas de aguacate obtuvo que el valor medio más alto lo registró el tratamiento micorrizas (40.2 unidades SPAD), mientras que Leal-Almanza et al. (2018) al evaluar Bacillus cereus, B. subtilis, Pseudomonas fluorescens y Trichoderma harzianum como promotores de crecimiento vegetal en el cultivo de papa Solanum tuberosum L., tampoco encontraron diferencias significativas. 
Lo anterior puede explicarse debido a que la aplicación de ácidos orgánicos junto con los microrganismos favorece su actividad en la rizosfera, además incrementa la arquitectura radicular y esto se refleja en una mayor asimilación de nutrientes en las plantas, y por ende un mayor contenido de clorofila (Badri y Vivanco, 2009; Zare-Maivan et al., 2017).

En cuanto a la altura de la planta en promedio el tratamiento Nutrisorb ${ }^{\circledR} \mathrm{L}+$ Biofit $^{\circledR}$ RTU presento en promedio $58.58 \mathrm{~cm}$ de longitud. Estos resultados coinciden con Canseco-Martínez et al. (2020) los cuales encontraron que aplicaciones de materia orgánica influyen directamente sobre el tamaño de plantas en comparación con el testigo sin aplicación, reportan que obtuvieron plantas de café (Coffea arabica L.) de mayor tamaño, debido a que al haber mayor cantidad de materia orgánica, existe también una mayor actividad microbiana y por ende mayor posibilidad de liberación de nutrientes que al ser aplicados al suelo continúan con el proceso de descomposición. Asimismo, Silveira et al. (2003) reporta un mayor desarrollo de follaje al realizar inoculaciones de micorrizas en aguacate.

En la variable longitud de la raíz el mejor tratamiento fue Nutrisorb ${ }^{\circledR} L+$ Biofit $^{\circledR}$ RTU, que presento una media de $63.23 \mathrm{~cm}$ mientras el testigo fue el de menor longitud. En plantas de aguacate, se ha demostrado los efectos de micorrizas en un mejor desarrollo radicular (Carreón-Abud et al., 2014) lo cual coincide con los resultados obtenidos. González y Fuentes (2017) mencionan que los microorganismos favorecen la producción de auxinas lo cual incrementa la longitud de las raíces.

En cuanto al peso seco de raíz la mezcla Nutrisorb ${ }^{\circledR} \mathrm{L}+$ Biofit $^{\circledR}$ RTU presento el mayor peso seco con $24.5 \mathrm{~g}$, seguido por los tratamientos de Glumix ${ }^{\circledR}$ y Nutrisob ${ }^{\circledR} \mathrm{L}+$ Mycoroot $^{\circledR}$. Barroetaveña y Rajchenberg (2003) y González y Fuentes (2017) reportan resultados similares al utilizar micorrizas, bacterias y Trichoderma encontrando un mayor número de raíces debido a la producción de fitohormonas como citoquininas lo que repercutió en el peso seco en plantas de pino (Pinus ponderosa Douglas ex C. Lawson), arroz (Oryza sativa L.) y lechuga (Lactuca sativa L.).

El porcentaje de micorrización fue influenciado por la aplicación de los ácidos orgánicos de la mezcla de Nutrisorb ${ }^{\circledR}$ L + Biofit $^{\circledR}$ RTU resultando el tratamiento con mayor porcentaje (73\%), seguido de Mycoroot $^{\circledR}$ y Glumix $^{\circledR}$ con 58 y $57 \%$ respectivamente (Figura 1).

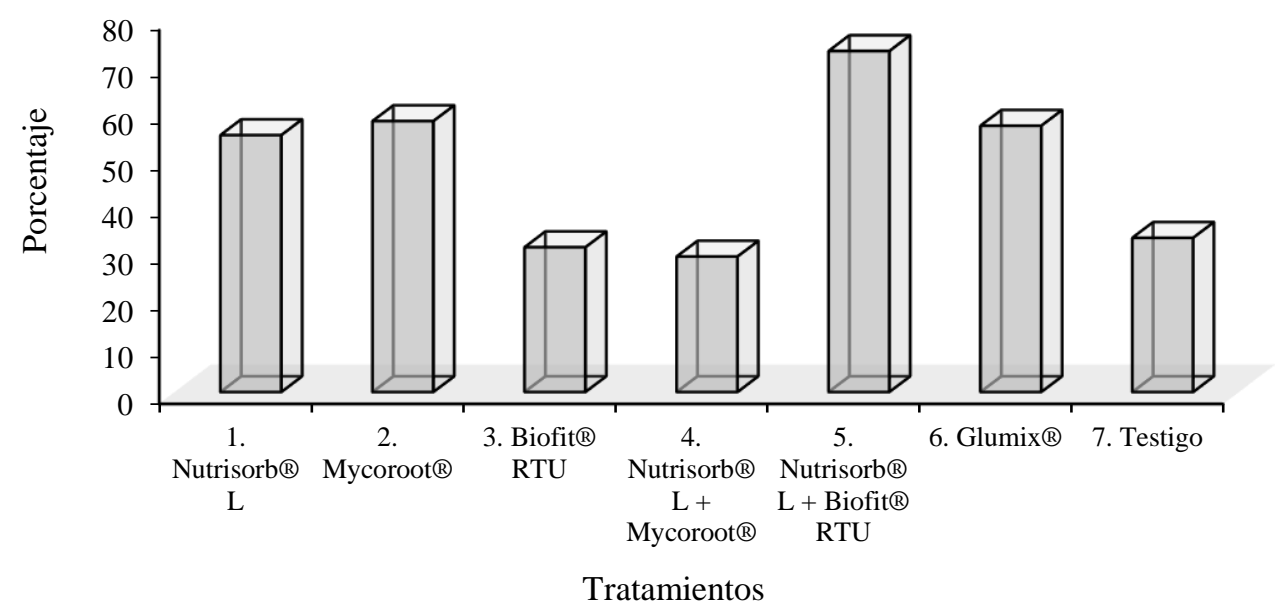

Figura 1. Efecto de bioestimulantes en el porcentaje de micorrización de plántulas de aguacate. 
Lo anterior coincide con Quiñones-Aguilar et al. (2014) quienes al incorporar fuentes de materia orgánica con micorrizas obtuvieron mayores porcentajes de micorrización en raíces de papayo (Carica papaya L.) en comparación con el testigo donde no se aplicaron. En vivero, se han tenido los mayores efectos en la implementación de la simbiosis de micorriza arbuscular, como una forma de mejorar la sanidad y el estado nutricional de las plantas, en la propagación de algunos frutales (Úsuga et al., 2008; Monticelli et al., 2000). Huang et al (2014); Dey y Sengupta (2020) mencionan que la presencia de sustancias orgánicas es vital para incrementar la actividad microbiana en la rizosfera, de tal manera que la planta se ve favorecida durante su desarrollo. Lo anterior, respalda estos resultados por lo que es factible la utilización de microrganismos y sustancias orgánicas dentro de programas de manejo de plántulas en viveros de aguacate.

\section{Conclusiones}

La aplicación de la mezcla de Nutrisorb ${ }^{\circledR} \mathrm{L}+$ Biofit $^{\circledR}$ RTU presenta el mayor efecto sobre las características de crecimiento y contenido de clorofila en las plántulas de aguacate injertadas con la variedad Hass en patrón criollo de la región por lo que es recomendable su uso para la producción comercial de plantas injertadas en vivero.

\section{Literatura citada}

Badri, D. V. and Vivanco, J. M. 2009. Regulation and function of root exudates. Plant, Cell Environ. 32:666-681.

Bais, H. P.; Weir, T. L.; Perry, L. G.; Gilroy, S. and Vivanco, J. M. 2006. The role of root exudates in rhizosphere interactions with plants and other organisms. Annu. Rev. Plant. Biol. 57:233-266.

Barroetaveña, C. y Rajchenberg, M. 2003. Las micorrizas y la producción de plántulas de Pinus ponderosa Dougl. ex Laws. en la Patagonia, Argentina. Bosque. 24(1):17-33.

Blasco, B.; Rios, J. J.; Cervilla, L. M.; Sánchez-Rodríguez, E.; Rubio-Wilhelmi, M. M.; Rosales, M. A.; Ruiz, J. M. and Romero, L. 2010. Photorespiration in lettuce plants (Lactuca sativa L.): Induced changes in response to iodine biofortification. J. Plant Growth Regul. 29(4):477-486.

Calvo, P.; Neklson, L. and Kloeper, J. W. 2014. Agricultural uses of plant biostimulants. Plant Soil. 383:3-41.

Campos-Rojas, E.; Ayala-Arreola, J.; Andrés-Agustín, J. y Espíndola-Barquera, M. de-la C. 2012. Propagación de aguacate. Servicio Nacional de Inspección y Certificación de Semillas (SNICS), Sistema Nacional de Recursos Fitogenéticos para la Alimentación y la Agricultura (SINAREFI), Universidad Autónoma Chapingo (UACH). 54 p.

Canseco-Martínez, D.; Villegas-Aparicio, Y.; Castañeda-Hidalgo, E.; Carrillo-Rodríguez, J.; Robles, C. y Santiago-Martínez, G. 2020. Respuesta de Coffea arabica L. a la aplicación de abonos orgánicos y biofertilizantes. Rev. Mex. Cien. Agríc. 11(6):1285-98.

Carreón-Abud, Y.; Aguirre, P. S.; Gavito, M. E.; Mendoza, S. D. J.; Juárez, C. R.; Martínez, T. M. y Trejo, A. D. 2014. Inoculación micorrízico arbuscular en portainjertos de plantas de aguacate $c v$ 'Hass' en viveros de Michoacán, México. Rev. Mex. Cien. Agríc. 5(5):847-857.

Dey, S. and Sengupta, S. 2020. Role of rhizospheric organic compounds on soil behavioral changes. Agriculture \& Food: eNewsletter. 2(5):221-225. 
Du Jardin, P. 2015. Plant biostimulants: definition, concept, main categories and regulation. Sci. Hortic. 196:3-14.

González, H. y Fuentes, N. 2017. Mecanismo de acción de cinco microorganismos promotores de crecimiento vegetal. Rev. Cienc. Agric. 34(1): 17-31.

Hayat, R.; Ali, S.; Amara, U.; Khalid, R. and Ahmed I. 2010. Soil beneficial bacteria and their role in plant growth promotion: a review. Ann Microbiol. 60:579-598.

Huang, X. F.; Chaparro, J. M.; Reardon, K. F.; Zhang, R.; Shen, Q. and Vivanco, J. M. 2014. Rhizosphere interactions: root exudates, microbes, and microbial communities. Botany. 92:267-275.

Koo, B. J.; Adriano, D. C.; Bolan, N. S. and Barton, C. D. 2005. Root exudates and microorganisms. In: Encyclopedia of Soils in the Environment. Hillel, D. (Ed.). Elsevier, Oxford, UK. 421-428 pp.

Leal-Almanza, J.; Gutiérrez-Coronado, M. A.; Castro-Espinoza, L.; Lares-Villa, F.; CortesJiménez, J. M. y Santos-Villalobos, S. 2018. Microorganismos promotores de crecimiento vegetal con yeso agrícola en papa (Solanum tuberosum L.) bajo casa sombra. Agrociencia. 52(8):1149-1159.

López-Bellido, R. J.; Shepherd, C. E. and Barraclough, P. B. 2004. Predicting post-anthesis N requirements of bread wheat with a Minolta SPAD meter. Eur. J. Agron. 20:313-320.

Monticelli, S.; Puppi, G. and Damiano, C. 2000. Effects of in vivo mycorrhization on micropropagated fruit tree rootstocks. Appl. Soil Ecol. 15(2):105-111.

Ortíz-Estrella, L. y Vázquez-Collado, I. 2008. Propagación. In: tecnología para producir aguacate en México. Coria-Ávalos, V. M. (Ed.). Libro técnico núm. 8. SAGARPA-INIFAP. $2^{\mathrm{a}}$. (Ed.). Uruapan, Michoacán, México. 8-16 pp.

Quiñones-Aguilar, E. E.; López-Pérez, L.; Hernández-Acosta, E.; Ferrera-Cerrato, R. y RincónEnríquez, G. 2014. Simbiosis micorrízica arbuscular y fuentes de materia orgánica en el crecimiento de Carica papaya L. Interciencia. 39(3):198-204.

Sakthiselvan, P.; Naveena, B. and Partha, N. 2014. Molecular characterization of a Xylanase producing fungus isolated from fouled soil. Braz. J. Microbiol. 45(4):1293-1302.

SIAP. 2020. Servicio de Información Agrícola y Pesquera. Boletín mensual de avance de la producción de aguacate. Secretaria de Agricultura y Desarrollo Rural. https://www.gob.mx/cms/uploads/attachment/file/539271/Avance_producci_n_de_

Aguacate_Enero_2020.pdf.

Silveira, S. V.; Souza, P. V.; Koller, O. C. and Schwarz, S. F. 2003. Elementos minerales y carbohidratos en plantones de aguacate 'Carmen' inoculados con micorrizas arbusculares. Proceedings V World Avocado Congress. 415-420 pp.

SAS. 2002. Statistical Analysis System. Statistical Analysis System. User's Guide v. 9.0. Cary NC., USA.

Úsuga, C. E.; Castañeda, D. A. y Franco, A. E. 2008. Multiplicación de hongos micorriza arbuscular (H.M.A) y efecto de la micorrización en plantas micropropagadas de banano (Musa AAA cv. Gran Enano) (Musaceae). Rev. Fac. Nal. Agr. Medellín. 61(1):4279-4290.

Zare-Maivan, H.; Khanpour-Ardestani, N. and Ghanati, F. 2017. Influence of mycorrhizal fungi on growth, chlorophyll content, and potassium and magnesium uptake in maize. J. Plant Nutr. 40(14):2026-2032. 